\title{
Continuous Clinical Assessment (CCA) in Baccalaureate Nursing Education: The Application of Action Research
}

\author{
Vico C L Chiang \\ PhD MHA GDMS BN RN FHKAN (Critical Care) FHKAN (Res \& Edu - Edu) \\ School of Nursing, The Hong Kong Polytechnic University
}

\begin{abstract}
The professional training of registered nurse $(\mathrm{RN})$ usually includes two major teaching and learning components, i.e. the theories and clinical practice. There is always room for improvement in teaching and learning and ways of evaluation of student competence regarding their clinical nursing skills. Students have indicated that too much focus has been placed on preparing them for the individual assessment tasks, and that the time to develop their skills more comprehensively to achieve better competence apart from the focus on task-orientated assessments is insufficient. The practice of clinical teachers has also been observed to be task-orientated with their tendency on focusing summative assessment rather than taking balanced perspective and efforts on the process of formative teaching and learning of the students. In order to optimize the efficacy of full formative and summative teaching and learning approach to develop the essential clinical skills, there is a vital need to vigorously promote and implement continuous clinical assessment (CCA) with the focus of explicit ongoing feedback of teachers to students during the process of clinical skills development, so that more effective and consolidating outcomes of students can be achieved and carried successfully over to their practice after graduation. The purpose of this paper is to discuss how the use of action research can actively promote the concept and teaching culture of CCA to the clinical teachers, and to empower them to apply CCA for better teaching and learning practice of the baccalaureate nursing students.
\end{abstract}

Keywords: nursing education, clinical teaching and learning, educational assessment, clinical competence 


\section{INTRODUCTION}

In order to comply with the assessment requirements and syllabus as mandated by various nursing registration councils, there are different teaching and assessment strategies used by nurse educators in different countries. For instance, clinical problem-based learning (Ehrenberg \& Haggblom, 2007; Oja, 2011) are applied to develop and evaluate students' competence during their clinical practica when they practise and learn different nursing skills and knowledge. On receiving feedback from many students and clinical teachers, there is usually room for improvement of the teaching and learning activities and the evaluation of student competence regarding the essential clinical nursing skills, e.g. aseptic technique (AT), administration of medication (AOM), and the assessment and implementation of competent holistic care for patients. From years of experience in nursing education, the author has observed that students also indicate too much focus is placed on preparing them for the individual assessment tasks, and that the time for them to develop their skills competence more comprehensively to achieve better performance apart from the task per se is insufficient. For example, even after passing the AT and AOM tests, many students are observed having the need to reinforce their skills at a later stage of their study. Although the teaching and learning of clinical skills demands a tremendous administrative load, such feedback induces a concern that the students can be taught and learn better for their clinical skills so that higher competence can be carried successfully over to their practice after graduation. Furthermore, as pointed out by Harlen and Deakin (2002), the focus of clinical teachers is found to be over-pragmatic and tend to be result-orientated towards summative assessment rather than the balanced effort with formative teaching and learning of the students. In order to optimize efficacy of a full formative and summative teaching and learning approach to develop essential clinical skills, there is a vital need to vigorously promote and implement continuous clinical assessment (CCA) with the altered focus on explicit ongoing feedback of teachers to students during the process of clinical skills development, so that more effective and consolidating outcomes of students can be achieved.

\section{The Concept and Practice of Continuous Clinical Assessment (CCA)}

Continuous clinical assessment has been used in nursing education since the 1970s in UK to profiling individual student's performance till the end of a particular course in order to assess the nursing competence (Chambers, 1998; Skelton, 1989). It involves the collection of evidence and decision making by the teachers in respect to experiential learning (Kolb, 1984) from clinical practice, multiple assessment methods, formative process that guides learning, and summative assessment of the nursing students (Lankshear \& Nicklin, 2000). It is used to evaluate students' competence in the provision of holistic nursing care through integration and application of learned knowledge and skills with the critical thinking to problem-solve.

In CCA, clinical teachers recognize students' strengths in learning, facilitate them to identify their learning issues, keep track of their intended learning outcomes (ILO) being developed and assessed, and guide them to improve their performance over a process of time with the fairest ongoing assessment of their performance in each stage of the clinical practice (Ellington \& Earl, 1997; Sweeney, 1989). Students are provided with continuous feedback on their performance (Oermann \& Gaberson, 2009) and encouraged to become more self-critical and reflective in improving their clinical skills. With the continuous 
monitoring of student performance, CCA can provide early warning signs of students who are having problems with their clinical practice. Hence, clinical teachers in CCA proactively and progressively facilitate students to achieve the required level of competence to the very end. It is believed that the model of CCA can further substantiate the teaching and learning of clinical skills and competence for nursing freshmen till the end of their study.

The implementation of CCA relied heavily on a portfolio or profiling approach (Sweeney, 1989) and such assessment is consistent with the competency approach of pedagogy (Spence \& El-Ansari, 2004). Within this continuous profiling process, clinical practice in the laboratory and real practice settings under CCA are the fundamental measures of teaching and learning of students. The CCA allows nursing students to continuously learn, integrate and apply nursing knowledge and skills through their actual experiences in the simulated and real environments. With respect to the experiential learning, Kolb (Kolb, 1984) theorized that the learning of students occurred through the cyclic process of 1) gaining concrete experience from clinical practice, 2) followed by reflective observation and 3) abstract conceptualization, and 4) subsequently the active experimentation that deepens such learning for another experiential learning cycle restarting from the gaining of further concrete experience. In the scope of Kolb's theory, the need to effectively evaluate clinical competence and develop nursing students through a continuous process as CCA with experiential learning is essential.

\section{LITERATURE REVIEW}

A review of the nursing literature indicated that CCA is usually not the specific term used, but 'continuous assessment', regarding the desirable continuous clinical teaching and learning of students. Continuous assessment is an approach of continuous teaching and learning through the use of portfolio and it is one way of CCA. There has been a majority of qualitative studies in this area. After some application of CCA in nursing education during the 1970s, continuous assessment of the learning of theories and practice in nursing education was officially recommended in 1983 by the United Kingdom Central Council for Nursing, Midwifery and Health Visiting (HKCC) (Stuart, 2007). For the professional requirement, measurement of competencies for a qualified nurse being developed through this process has to ensure various skills in managing patient care within the constantly changing professional and health care contexts (Stuart, 2007). Conceptually, Bedford, Phillips, Robinson \& Schostak (1993), Fraser, Murphy \& Worth-Butler (1997), Phillips Schostak \& Tyler (2000) and Nursing and Midwifery Council (2008) held that CCA was a cumulative process of teaching and learning facilitated by the clinical supervisors/ assessors, which could only be fairly performed over an unhurried period of time with good planning and communication with students. After continuous and sufficient learning opportunities in the clinical context were provided for the range of clinical experiences required to develop professional nursing competence, total learning of students can be accurately estimated (Stuart, 2007).

Quinn (1997) pointed out that episodic assessment that involved the tests of specific clinical skills at prearranged time was artificial and it lacked incorporation of the dynamic aspects of reality as a process for more realistic assessment of student's competence. Episodic assessment was also more stressful for students to take and it failed to represent the student's performance over a period of time. Therefore, CCA that concentrates on the assessment of practice as a process 
over a period of time ensures that no particular skills can be passed on the basis of 'once and for all' like those expected in the episodic assessment model (Nichlin \& Kenworthy, 2000). The implementation of CCA is a positive step forwards in these regards (Hanley \& Higgins, 2005) but in contrast to the argument of Quinn (1997), CCA has also been criticized on the grounds of subjectivity and variability among assessors (Aggleton, Allen \& Montgomery, 1987; Hanley \& Higgins, 2005; McMullan et al., 2003; Neary, 2001; While, 1994). Girot (1993) also identified the similar problem of assuring objectivity of the assessors and establishing validity and reliability of the assessment over an extended period. For instance, it was difficult to develop valid and reliable tools to assess clinical competence (Chambers, 1998; Hanley \& Higgins, 2005). Although assessment guidelines are usually available, individual assessors may have different perceptions of competence from their own standpoints, based on their own values, beliefs and experiences. And during the early period of assessment, desirable personal traits of students such as appearance and personality were often judged subjectively by the assessors. According to Chambers (1998), this may have been related to the issue that there is little consensus of what is meant by the term 'competence' when applied to clinical nursing practice, which makes the clinical practice assessment mainly a subjective process.

Nevertheless, Kenworthy and Nicklin (1989) described CCA as 'snapshots' of practice as opposed to the single snapshot of prearranged practical assessment previously described as episodic (Quinn, 1995). The facet of CCA on self-assessment (for instance the reflection and profile assessment) of students was at least a more efficient and effective learning tools that allowed the students to identify their own strengths and weaknesses (Chambers, 1998). With those contrasting views and findings, better development of CCA regarding the objectivity of assessors, validity and reliability in assessing clinical competence of students remains an area for further research and development. Empirical studies in the nursing literature that explored or evaluated the process of CCA are sparse. The studies were usually qualitative or mixed by method (Al Kadri, Al-Moamary \& van der Vleuten, 2009; McMullan, 2008; Neary, 1999; Neary, 2001; Spence \& ElAnsari, 2004; Thome, Hovenberg \& Edgren, 2006; Web et al., 2003) but one survey (McMullan, 2006).

Out of the literature, it is clear that in order to succeed in the implementation of CCA for clinical teaching and learning of nursing students, there are essential aspects for the nursing educators and researchers to master. There remains gaps for further studies to bridge and they include, 1) reinforcing the accountability and a culture of teaching practice of the clinical assessors / teachers in CCA to authentically practise continuous assessment of student's clinical competence which is founded on the optimal levels at the time and context the students can achieve, and 2) determining the clinical progress of student's clinical learning and competence with a more valid and reliable way of assessment. Therefore, research is needed to actively promote the concept and teaching culture of CCA to the clinical teachers and empower them to apply it for better teaching and learning practice of the baccalaureate nursing students. The goals of CCA for better continuous assessment, feedback and outcomes of student's clinical competence, as well as better engagement and application of CCA by the clinical teachers can be examined. Recommendations can be made for further development.

\section{Implications for Further Studies}

With the key purpose to promote, apply and examine the development of CCA, related research may utilize the action research design (Hinchey, 2008) with focus group 
activities and surveys to introduce and continuously develop CCA through the implementation of such teaching and teaching approach, and the empowerment of clinical teachers involved. Action research fits the purpose and questions 1) to explore the experiences of clinical teachers and outcomes of the CCA process for clinical practica of the baccalaureate nursing programmes, and 2) to explore and understand the perceptions of clinical teachers and students in nursing about the characteristics of effective clinical teachers; so that, a) the goals of CCA for better continuous feedback and assessment of student's clinical competence, and b) better engagement and application of CCA by the clinical teachers for further development of CCA can be achieved.

Action research involves an empowering spiral process that takes into play the key elements of emic (insider) views of clinical teachers and their active role as the change agents during the CCA, and the inputs of researcher's etic (outsider) views (Streubert \& Carpenter, 2011). The key benefits of action research includes the opportunities for and maneuvers of the participating teachers in such exercise to actively and continuously taking part and reflect, and being empowered, in the process and narrow the theory and practice gap in teaching and learning (Mitchell, Reilly \& Logue, 2009). Hence, this is a democratic cyclic process of back and forth interactions among the participating teachers with the researchers (Streubert \& Carpenter, 2011). The process is a continuous spiral of planning, action, monitoring, and evaluation and feedback by reflection (Kemmis, 1983) for the participating teachers to re-design the plan and implementation of CCA (Figure 1). The subsequent cycles continue until the end of the target period. This proposal is the initial planning stage for the following cycles to come. Reflection is a key element for the success of this process (Fisher, Bennett-Levy \& Irwin, 2003) in addition

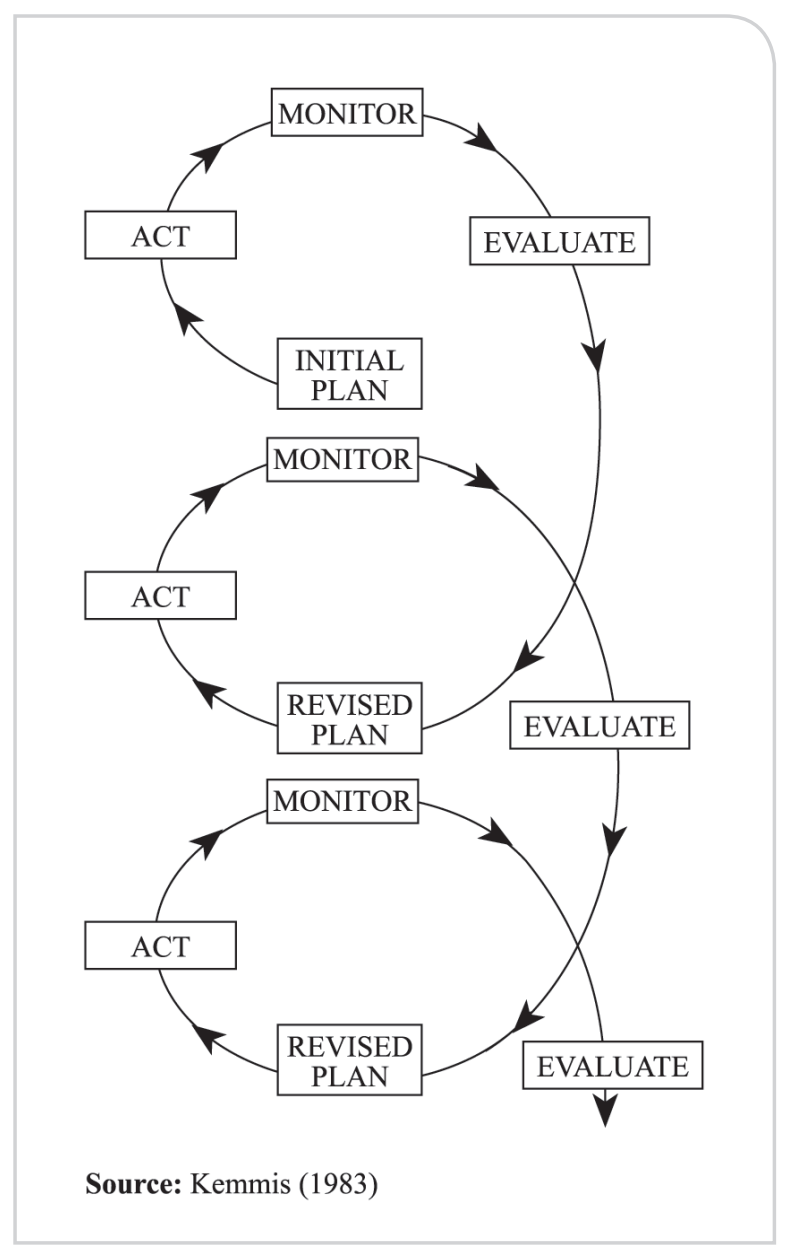

Figure 1. The spiral process of action research

to the democratic empowerment (Streubert \& Carpenter, 2011).

\section{METHODS}

\section{Participants}

As in qualitative research, action research can utilize a purposeful sampling strategy in which the most informative informants will be invited to participate in the study (Polit $\&$ Beck, 2010). In this regard, the richest information potential participants for such a study will provide (Patton, 1990) are all the clinical teachers and nursing students 
who will directly involve in the teaching and learning through CCA during the particular baccalaureate nursing programme. The nursing programme may have a training focus on medical / surgical and mental health nursing, and hence the majority of clinical practica is arranged for these areas of nursing practice. Clinical teachers may be recruited to the study from CCA briefing session prior to the first student's clinical placement. And students may be recruited during their clinical practicum briefing sessions. These briefings are routine sessions usually provided to teachers and students before commencement of a new semester. Recruited clinical teachers may take part in a series of surveys and focus group activities during the spiral process of action research, while the students participate only in a series of surveys and clinical teacher evaluation during the process. It is deemed sufficient to have the CCA process promoted, implemented, evaluated and further developed with the action research process for a total period of two years.

\section{The CCA Programme}

In order to implement the CCA, there may be three teaching and learning activities for students, 1) skill reinforcement workshop (SRW), 2) clinical portfolio (CP), and 3) case study report (CSR), etc; and the activity of CCA moderation through focus groups for clinical teachers.

\section{CCA Moderation}

Under the CCA for clinical skills development like those of AT / AOM, there will be no more one-off final tests but continuously (formatively) performed based on the feedback from the teachers, until a time when the summative decision is needed. Once the students have completed the SRW, they will be continuously assessed and feedback within the CCA process by their supervising clinical teachers for evaluation and ongoing development of their clinical skills competence in the relevant clinical settings. The CCA moderation process aims to empower the clinical teachers to effectively and successfully master such continuous teaching and learning process, and this will be done through a series of focus groups within the spiral process (through a number of cycles) of the action research.

During the focus groups sessions the clinical teachers will be encouraged and reminded to keep a reflective journal for their teaching and learning with students during the CCA. Reflection is an essential activity for the participating teachers to develop their education practice (Mitchell, Reilly \& Logue, 2009), in particular in the focus groups when they can observe the best from other teachers and engage in reflective dialogues with them (Connelly \& Clandinin, 1995). The focus group sessions will serve as the best occasions for them to engage in such constructive dialogues for their own development as well as the CCA.

Rigor of the CCA will be assured by the qualitative approach of trustworthiness as proposed by Webb et al. (2003) and Shenton (2004). Using such an approach can demonstrate that the evidence contained within and expressed through the outcomes and documentation of CCA is sufficiently rigorous to indicate student competence.

\section{Data Collection Procedure}

After clear explanation and informed consent obtained from the potential participants, data will be corrected in a series through a set of survey instruments with the clinical teachers and students, and feedback through numerous 
focus groups with the teachers. Activities in the focus groups will target at individual and collective reflection and a team process for the clinical teachers to communicate, evaluate, and making further recommendations for the continuous implementation of CCA. Apart from the focus groups, additional seminars or briefing sessions will be organized as needed throughout the process to consolidate the utilization of CCA and its ongoing development across the study years. It is critical for the principal investigator and his / her associate/s to ensure that the process is a continuous one with regular feedback for the improvement and refinement of CCA, and a process which involves and empowers the clinical teachers. Clinical teachers are the key parties in the entire dynamic and interactive process with other teachers in the team (Streubert \& Carpenter, 2011).

As mentioned above, the entire project may probably take two years for the cyclic / spiral process of action research to complete. Changes are therefore anticipated when all those planned activities and interactions of the clinical teachers come into play during the dynamic action research process (Lewin, 1946). There are quantitative and qualitative data collections and analyses in the study. The concurrent results generated will be used in the subsequent focus groups of clinical teachers to enhance the process that promotes CCA.

\section{Recommended Survey Instruments and Data Collection Methods}

\section{Demographic Data Sheet}

First of all, demographic information will be collected from all the participants. Clinical teachers and students who provide consent to take part in the study will be asked to anonymously complete a demographic data sheet.

\section{Nursing Clinical Teacher Effectiveness Inventory (NCTEI)}

The Nursing Clinical Teacher Effectiveness Inventory (NCTEI) may be used to identify the statuses and evaluate any changes of the effective characteristics of clinical teachers during the CCA process from the students' and teachers' own perspectives. The NCTEI is a 48 item checklist developed by Knox and Mogan (1985) and Mogan \& Knox (1987), to investigate the specific characteristics differentiating 'best' from 'worst' clinical teachers according to the perceptions of clinical teachers and their undergraduate students from different university schools of nursing in the United States and Canada. Apart from the North America (Mogan \& Knox, 1987; Nehring, 1990), the scale was repeatedly used in different populations including Hong Kong (Li, 1997), Greece (Kotzabassaki et al., 1997) and Australia (Lee, Cholowski \& Williams, 2002). From the study by Mogan and Knox (1987), the NCTEI has a good internal consistency (Cronbach's Alpha ranged from 0.79 - 0.92), is stable over time (test-retest scores at 4-week intervals ranged from the Pearson's correlations of $r=0.76-0.93)$, and considered to have content and face validity. The NCTEI is openly available from cIRcle of the University of British Columbia for public access on the web (https://circle.ubc.ca/). The checklist describes discrete clinical teacher characteristics which are clustered into five subscales of teaching ability, interpersonal relationships, personal characteristics, nursing competence, and evaluation. Respondents indicate on a seven-point Likert scale how descriptive of a specific characteristic is of a clinical teacher $(1=$ not at all important to $7=$ very important). Higher scores imply more positive characteristics in clinical teaching.

In the action research, the results of NCTEI will serve as a guide for clinical teachers' improvement. Results 
of the NCTEI obtained from students and among the teachers themselves will be generated across the 2-year period of CCA of the study. Since the beginning of study when NCTEI data were collected, the anonymous results will be made available to teachers in the focus groups at different time points for them to collaboratively review and reflect upon, while individual results will be returned confidentially. Clinical teachers can therefore examine their own instructional behaviours in the light of students' as well as their peer's perceptions for own development or improvement in clinical teaching. They can also try to incorporate those identified effective behaviours and avoid those ineffective ones in their clinical teaching repertoire. To the minimum, those ineffective behaviours being identified can alert the teachers about their potential problematic areas of clinical teaching (MacDonaldClarkson, 1986).

\section{Clinical Evaluation for Clinical Practicum of Entry / Intermediate / Exit Level (CE-CP-L)}

The CE-CP-L is specific to individual baccalaureate nursing programmes and usually developed based on the feedback from teachers and students regarding their previous experiences of clinical teaching and learning. Face validity is usually established by a panel of experts in the respective schools and the tool is tested for feasibility with good feedback from clinical teachers for its strong capacity to explicitly highlight both the formative feedback and summative assessment to students based on their status at the entry, intermediate, and exit level. For examples, the CE-CP-L may have three clinical domains of general competencies, application of nursing process, and professional qualities. And each of these essential domains can be formatively and summatively evaluated with a 4-point Likert scale (below, attained, above, or outstanding of the expected level) according to the appropriate set of criteria by different levels. The CE-CP-L aids the practical application of CCA by the clinical teachers and documentation of the progress and outcomes of students for further learning and development purposes. Further psychometrics investigation is desired for this evaluation tool though it is not the key purpose of the action research project. Reliability of this tool may be analyzed with the data collected during the periods of action research cycles.

\section{Focus Group Activities}

An initial briefing session and a series of focus groups will be conducted with the clinical teachers in this action research to promote and achieve the best development and outcomes of CCA as well as the clinical teaching practice within such model. There will be cycles of focus group activities in the action research process. Each group may have 6-8 members (Thomas et al., 1992). The interactive dialogues and reflective process involved in the focus groups are the essential elements to empower the clinical teachers to discuss and reflect on their clinical teaching for individual development (Streubert \& Carpenter, 2011), and that the feedback or any constructive comments for ongoing improvement and development of the CCA model can also be made. Conversation and discussion in the focus groups will be moderated by the researcher/ s each time to engage the group members. Essential activities and processes of the focus groups for all members to participate aim at developing the 'accountability' to CCA through 'mutual support', which is emphasized on the action research model of Fisher, Bennett-Levy and Irwin (2003). These are the works of empowerment to the group members as teachers for CCA, and reflection and sharing are the crucial exercise for all members to perform through the activities like 'check-ins' to establishing mutual 
support for accountability (Fraser et al., 1997) in the spiral process.

Additional sessions may be added when necessary for any ad hoc issues that the teaching team need to promptly explore and resolve during the CCA process. During the focus groups, audio recording will be made to capture all the conversation for subsequent verbatim transcription and qualitative analysis. Such analysis is constantly compared (Glaser \& Strauss, 1967), which aims to identify major categories regarding the clinical teaching of CCA. With the outputs of NCTEI obtained regularly from both the clinical teachers and students in a series of time throughout the process, sharing and discussion of the clinical teachers' individual reflection of their clinical teaching in CCA will be facilitated during the focus groups. Qualitative analyses of the verbatim data generated from focus groups would have been performed and the results discussed in the subsequent focus groups so that the spiral process of action research and the purpose of empowering clinical teachers can be achieved based on the ongoing interactive exchanges.

\section{Data Analyses}

The quantitative data will be entered into a spreadsheet for statistical analysis. The principal researcher will check the dataset against the original data collection sheets of the completed questionnaires. Descriptive statistics will be generated for the demographics and any significant differences considered for further data analyses.

With the scores of baseline NCTEI and subsequent data collected during the course of CCA and the action research process obtained from students and clinical teachers, analysis of variance, e.g. repeated measures ANOVA, will be performed to identify any changes of teacher's effective teaching behaviours in an ongoing fashion along the 2-year period of the study. Variability and reliability of the NCTEI scores obtained from students and teachers over time will be examined with the inter-class correlation (ICC). The clinical levels of competence obtained by students as recorded in the CE-CP-L will be compared statistically with those of the corresponding results from the previous classes of students who commenced their study when the CCA was not implemented. Within group comparisons will also be done with the data being collected from the participating class of students over the study period. This will demonstrate any significant differences within the class of CCA, as well as between the two classes with and without the CCA. The level of significance of all statistical tests will be set at $\leq 0.05$.

Qualitative content analysis with an inductive approach (Elo \& Kyngas, 2007; Mayan, 2001; Sandelowski, 2000) will be used to analyze the data. With this approach, transcripts generated from all the focus groups are analyzed through the process of coding to identify main categories. A computer-aided qualitative data analysis software (CAQDAS) NVivo may be used to aid the management of data and the analytic process. Again, the ongoing results obtained from NCTEI and qualitative analysis are very important to provide the basis for focus group discussion and reflection in this project as the means for exploring and developing effectiveness of clinical nursing education through CCA. The series of focus groups in this action research target to promote, empower, apply, and improve the teaching of clinical teachers who work under the CCA model for the best competence development of baccalaureate nursing students. 


\section{LONG-TERM IMPACT \& CONCLUSION}

The needs of diverse populations in the society have rapidly evolved and developed under the highly technical and advanced health care environment. The complexity and need for efficient and effective clinical nursing education has increased as it is being challenged by such increasingly complex environment. In addition to the theoretical components, clinical teachers in nursing must be empowered and equipped to enable successful clinical education for appropriate knowledge and competent skills that meet the needs of patients and the society (Hsu, 2006). But it has been observed that clinical teachers in general tend to be result-orientated and target the summative assessment of student competence rather than a good balance with the process of continuous teaching and learning (Harlen \& Deakin, 2002).

Through the action research process, clinical teachers will be facilitated and empowered to participate in the change of teaching culture for CCA and develop more efficient and effective teaching and learning practice. In turn the good professional nursing identity and competent clinical skills of students for now and in the future will be better assured. Results generated from the action research study will also add to the limited literature regarding implementation and outcomes of CCA in a preregistration undergraduate nursing programme, so that further recommendations and development can be made. In particular when there is a paucity of studies which investigate the relationship of effective clinical education and patient outcomes (Reilly, 2007), the results generated from action research will inform future empirical studies that verify which components of education are accountable for what outcomes in the context of clinical nursing education. Identification and establishment of an effective
CCA model are the beginning steps to path this kind of correlation studies. Other appropriate recommendations will also be made for further development of CCA. In this connection, more nurse educators may consider implementing pilot translational projects to apply the CCA model for better clinical teaching and learning of the preregistration undergraduate nursing students.

In summary, with the need for better clinical nursing education and the gaps identified from literature, clinical teachers can be empowered through action research to apply CCA for better teaching and learning practice of nursing students in the baccalaureate programmes.

\section{Acknowledgment}

The author of this article appreciates the advice provided by Professor Wong Kam Yuet Frances, and the inspiration of Mr Kwan Yiu Cho, during the conception of an action research study for the promotion and development of CCA.

\section{REFERENCES}

Aggleton, P., Allen, M., \& Montgomery, S. (1987). Developing a system for the continuous assessment of practical nursing skills. Nurse Education Today, 7(4), 158-64.

Al Kadri, H. M. F., Al-Moamary, M. S., \& van der Vleuten, C. (2009). Students' and teachers' perceptions of clinical assessment program: A qualitative study in a PBL curriculum. BMC Research Notes, 2, Available: http://www.biomedcentral.com/1756-0500/2/263

Bedford, H., Phillips, T., Robinson, J., \& Schostak, J. (1993). Assessment of competencies in nursing and midwifery education and training. London: The English National Board for Nursing, Midwifery and 
Health Visiting.

Chambers, M. A. (1998). Some issues in the assessment of clinical practice: A review of the literature. Journal of Clinical Nursing, 7, 201-208.

Connelly, F., \& Clandinin, D. (1995). Teachers' professional knowledge landscapes: Secret, sacred, and cover stories. In D. Clandinin, \& F. Connelly (eds.), Teachers' professional knowledge landscapes (pp. 3-15). New York: Teachers College Press.

Ehrenberg, A. C., \& Haggblom, M. (2007). Problem-based learning in clinical nursing education: Integrating theory and practice. Nurse Education in Practice, 7, 67-74. doi: 10.1016/j.nepr.2006.04.005

Ellington, H., \& Earl, S. (1997). Making effective use of continuous assessment and portfolios. Retrieved August 2, 2011, from http://www.nalanda.nitc.ac.in/ misc/general/ciced/Ch25. html

Elo, S., \& Kyngas, H. (2007). The qualitative content analysis process. Journal of Advanced Nursing, 62(1), 107-115.

Fisher, K., Bennett-Levy, J., \& Irwin, R. (2003). What a GAS! Action research as a peer support process for postgraduate students. uliBASE, Nov. Retrieved Sept 24, 2011, from http://ultibase.rmit.edu.au/Articles/ nov03/fisher.pdf

Fraser, D., Murphy, R., \& Worth-Butler, M. (1997). An outcome evaluation of the effectiveness of preregistration midwifery programmes of education. London: The English National Board for Nursing, Midwifery and Health Visiting.

Girot, E. (1993). Assessment of competence in clinical practice: A review of the literature. Nurse Education Today, 13, 83-90.

Glaser, B. G., \& Strauss, A. L. (1967). The discovery of grounded theory: Strategies for qualitative research. New York: Aldine De Gruyter.

Hanley, E., \& Higgins, A. (2005). Assessment of clinical practice in intensive care: A review of the literature. Intensive and Critical Care Nursing, 21, 268-275.
Harlen, W., \& Deakin, C. R. (2002). A systematic review of the impact of summative assessment and tests on students' motivation for learning. In Research Evidence in Education Library. London: EPPI-Centre, Social Science Research Unit, Institute of Education, University of London. Available: http://eppi.ioe.ac.uk/ $\mathrm{cms} /$ Default.aspx?tabid=108\& language=en-US

Hinchey, P. H. (2008). Action research primer. New York, NY: Peter Lang.

Hsu, L. L. (2006). An analysis of clinical teacher behaviour in a nursing practicum in Taiwan. Journal of Clinical Nursing, 15(5), 619-28.

Kemmis, S. (1983). Action Research. In D. S. Anderson, \& C. Blakers (eds.), Youth, transition and social research (pp.130-152). Canberra: Australian National University.

Kenworthy, N., \& Nicklin, P. (1989). Teaching and assessing in clinical practice: An experiential approach. London: Scutari.

Knox, J., \& Mogan, J. (1985). Important clinical teacher behaviours as perceived by University nursing faculty, students and graduates. Journal of Advanced Nursing, 10, 25-30.

Kolb, D. A. (1984). Experiential learning: Experience as the source of learning and development. New Jersey: Prentice-Hall.

Kotzabassaki, S., Panou, M., Dimou, F., Karabagli, A., Koutsopoulou, B., Ikonomou, U. (1997). Nursing students' and faculty's perceptions of the characteristics of "best" and "worst" clinical teachers: A replication study. Journal of Advanced Nursing, 26(4), 817-24.

Lankshear, A., \& Nicklin, P. (2000). Methods of assessment. In P. J. Nicklin, \& N. Kenworthy, Teaching \& assessing in nursing practice (pp.120-138). London: Bailliere Tindall.

Lee, W., Cholowski, K., \& Williams, A. K. (2002). Nursing students' and clinical educators' perceptions of characteristics of effective clinical educators in an Australian university school of nursing. Journal of Advanced Nursing, 39(5), 412-20. 
Lewin, K. (1946). Action research and minority problems. In G. W. Lewin (ed.), Resolving social conflicts: Selected papers on group dynamics by Kurt Lewin (pp.201-216). New York: Harper and Row.

Li, M. K. (1997). Perceptions of effective clinical teaching behaviours in a hospital-based nurse training programme. Journal of Advanced Nursing, 26(6), 1252-1261.

MacDonald-Clarkson, C. M. (1986). Characteristics of effective and ineffective clinical teachers in nursing as perceived by students and faculty. Unpublished Master of Science Dissertation. School of Nursing, The University of British Columbia. Retrieved September 18, 2011, from https://circle.ubc.ca/ bitstream/handle/2429/26005/ UBC_1986_A6_7\%20 M32_5.pdf

Mayan, M. J. (2001). An introduction to qualitative methods: A training module for students and professionals. Edmonton: International Institute for Qualitative Methodology.

McMullan, M. (2006). Students' perceptions on the use of portfolios in pre-registration nursing education: A questionnaire Survey. International Journal of Nursing Studies, 43(3), 333-343.

McMullan, M. (2008). Using portfolios for clinical practice learning and assessment: The pre-registration nursing student's perspective. Nurse Education Today, 28, 873-879.

McMullan, M., Endacott, R., Gray, M. A., Jasper, M., Miller, C. M. L., Scholes, J., \& Webb, C. (2003). Portfolios and assessment of competence: A review of the literature. Journal of Advanced Nursing, 41(3), 283-294.

Mitchell, S. N., Reilly, R. C., \& Logue, M. E. (2009). Benefits of collaborative action research for the beginning teacher. Teaching and Teacher Education, 25(2), 344-349.

Mogan, J., \& Knox, J. (1987). Characteristics of best and worse clinical teachesr as perceived by university faculty and students. Journal of Advanced Nursing, 12(3), 331-337.
Neary, M. (1999). Preparing assessors for continuous assessment. Nursing Standard, 13(18), 41-47.

Neary, M. (2001). Responsive assessment: assessing student nurses' clinical competence. Nurse Education Today, 21, 3-17.

Nehring, V. (1990). Nursing Clinical Teacher Effectiveness Inventory: A replication study of the characteristics of "best" and "worst" clinical teachers as perceived by nursing faculty and students. Journal of Advanced Nursing, 15(8), 934-940.

Nichlin, P. J., \& Kenworthy, N. (2000). Teaching and assessing in nursing practice: An experimental approach (4th ed.). London: Bailliere Tindall.

Nursing and Midwifery Council (NMC) (2008). Standard to support learning and assessment in practice. London: NMC. Retrieved September 5, 2011, from, http://www.nmc-uk.org/Documents/Standards/nmcS tandardsToSupportLearningAndAssessmentInPracti ce.pdf

Oermann, M. H., \& Gaberson, K. B. (2009). Evaluation and testing in nursing education (3rd ed.). New York: Springer.

Oja, K. J. (2011). Using problem-based learning in the clinical setting to improve nursing students' critical thinking: An evidence review. The Journal of Nursing Education, 50(3), 145-151. doi: 10.3928/0148483420101230-10

Patton, M. Q. (1990). Qualitative evaluation and research methods (2nd ed.). Newbury Park, California: Sage.

Phillips, T., Schostak, J., \& Tyler, J. (2000). Practice and assessment in nursing and midwifery: Doing it of real. London: The English National Board for Nursing, Midwifery and Health Visiting.

Polit, D. F., \& Beck, C. T. (2010). Essentials of nursing research: Appraising evidence for nursing practice (7th ed.). Philadelphia: Lippincott Williams \& Wilkins.

Quinn F.M. (1995). The Principles and Practice of Nurse Education (3rd ed.). London: Chapman and Hall.

Reilly, B. M. (2007). Inconvenient truths about effective clinical teaching. Lancet, 370, 705-711. 
Sandelowski, M. (2000). Whatever happened to qualitative description? Research in Nursing and Health, 23, 334-340.

Shenton, A. K. (2004). Strategies for ensuring trustworthiness in qualitative research projects. Education for Information, 22, 63-75.

Skelton, G. M. (1989). Assessment and profiling of clinical performance. In P. L. Bradshaw (ed.), Teaching and assessing in clinical nursing practice (pp.160-174). London: Prentice Hall.

Spence, W., \& El-Ansari, W. (2004). Portfolio assessment: practice teachers' early experience. Nurse Education Today, 24, 388-401.

Streubert, H. J., \& Carpenter, D. R. (2011). Qualitative research in nursing (5th ed.). Philadelphia: Lippincott Williams \& Wilkins.

Stuart, C. C. (2007). Assessment, supervision and support in clinical practice: A guide for nurses, midwives and other health professionals (2nd ed.). Philadelphia: Churchill Livingstone Elsevier.

Sweeney, J. F. (1989). Student profiling as a basis for continuous assessment of clinical progress during a registered mental nurse course (1988). Nurse Education Today, 9(4), 254-263.

Thomas, S., Steven, I., Browning, C., Dickens, E., Eckermann, L., Carey, L., \& Pollard, S. (1992). Focus groups in health research: A methodological review. Annual Review of Health Social Sciences, 2, 7-20.

Thome, G., Hovenberg, H., \& Edgren, G. (2006). Portfolio as a method for continuous assessment in an undergraduate health education programme. Medical Teacher, 28(6), e171-e176.

Webb, C., Endacott, R., Gray, M. A., Jasper, M. A., McMullan, M., \& Scholes, J. (2003). Evaluating portfolio assessment systems: what are the appropriate criteria? Nurse Education Today, 23, 600609.

While, A. (1994). Competence versus performance: which is more important? Journal of Advanced Nursing, 20(3), 525-31. 
